\title{
An Efficient Method to Compute Pressure on Blades of Hydraulic Machines
}

\author{
Vyacheslav Kalashnikov, Ph.D. ${ }^{1,2,3}$, Nataliya Kalashnykova, Ph.D. ${ }^{4}$, and Felipe J. Castillo-Pérez, Ph.D. ${ }^{1}$ \\ ${ }^{1}$ Tecnológico de Monterrey (ITESM), Campus Monterrey, Mexico, kalash@itesm.mx, slavkamx@gmail.com \\ ${ }^{2}$ Central Economics and Mathematics Institute (CEMI), Russia, slavkamx@mail.ru \\ ${ }^{3}$ Sumy State University, Ukraine, mxkalash@yahoo.de \\ ${ }^{4}$ Universidad Autónoma de Nuevo León (UANL), Mexico, nkalash2009@gmail.com
}

\begin{abstract}
In this paper we use the method of discrete hydrodynamic singularity and a condition on the velocity normal component at the flow's boundary in order to reduce the problem of ideal liquid flow around arbitrarily positioned bodies to the solution of an appropriate linear equations with a block matrix structure. Having applied discretization in such a way that the resulting system's matrix boasts an implicit diagonal dominance, one can establish the convergence of a block Jacobi iteration method to the exact solution of the system. The algorithm's efficiency is illustrated by a sample computing of hydrodynamic pressure on the ring blades of a hydraulic turbine.
\end{abstract}

Keywords - Method of discrete hydrodynamic singularity, the implicit diagonal dominance, hydrodynamic charges.

Digital Object Identifier (DOI): http://dx.doi.org/10.18687/LACCEI2015.1.1.109

ISBN: 13 978-0-9822896-8-6

ISSN: $2414-6668$

13 $^{\text {th }}$ LACCEI Annual International Conference: "Engineering Education Facing the Grand Challenges, What Are We Doing?" July 29-31, 2015, Santo Domingo, Dominican Republic

ISBN: 13 978-0-9822896-8-6

ISSN: $2414-6668$

DOI: $\underline{\text { http://dx.doi.org/10.18687/LACCEI2015.1.1.109 }}$ 


\title{
An Efficient Method to Compute Pressure on Blades of Hydraulic Machines
}

\author{
Vyacheslav Kalashnikov, Ph.D. ${ }^{1,2,3}$, Nataliya Kalashnykova, Ph.D. ${ }^{4}$, and Felipe J. Castillo-Pérez, Ph.D. ${ }^{1}$ \\ ${ }^{1}$ Tecnológico de Monterrey (ITESM), Campus Monterrey, Mexico, kalash@itesm.mx, slavkamx@gmail.com \\ ${ }^{2}$ Central Economics and Mathematics Institute (CEMI), Russia, slavkamx@mail.ru \\ ${ }^{3}$ Sumy State University, Ukraine, mxkalash@yahoo.de \\ ${ }^{4}$ Universidad Autónoma de Nuevo León (UANL), Mexico, nkalash2009@gmail.com
}

\begin{abstract}
In this paper we use the method of discrete hydrodynamic singularity and a condition on the velocity normal component at the flow's boundary in order to reduce the problem of ideal liquid flow around arbitrarily positioned bodies to the solution of an appropriate linear equations with a block matrix structure. Having applied discretization in such a way that the resulting system's matrix boasts an implicit diagonal dominance, one can establish the convergence of a block Jacobi iteration method to the exact solution of the system. The algorithm's efficiency is illustrated by a sample computing of hydrodynamic pressure on the ring blades of a hydraulic turbine.

Keywords-- Method of discrete hydrodynamic singularity, the implicit diagonal dominance, hydrodynamic charges.
\end{abstract}

\section{INTRODUCTION}

Nowadays, before developing and assembling hydraulic machines, the manufacturers seek to elevate their efficiency by making use of the advanced theory and computational experiments [1]. Thus obtained results are extremely handy for the further improvement of various properties of the produced machines, such as their power efficiency, cavitation immunity, compression charges, as well as the parameters of the nonstationary flow around a rotor's main body. The latter means that the quite expensive physical and technical experiments related to the hydraulic machines could be replaced with much cheaper numerical tests, which, in turn, would accelerate the achievement of the desired characteristics of the newly created machine samples.

Therefore, the quality requirements to the numerical results, as well as the challenges related to the numerical solution of the problem of flow around solid bodies have attracted a lot of attention of many researchers worldwide since long. Solutions of some problems related to those examined here can be found are in [1] - [15], [18], to mention only few.

It is well-known that to study a potential flow of an incompressible fluid around a rotor, one needs to solve an appropriate linear system obtained by a discretization method. The system's dimension depends upon the number of discrete nodes selected on the profile. Computational experience shows that in order to guarantee any acceptable precision the number of computation nodes must quite large. The latter leads to considerable challenges to the numerical implementation of the existing algorithms, even when cut-ofthe-edge computers are used. The other difficulties that arise include, for instance: $(i)$ the problem of flow around the stator columns and the regulating ring blades of a hydraulic turbine positioned in a volute chamber; (ii) the problem of flow around profile grids that is non-uniform at the inlet, and (iii) the problem of hydrodynamic interaction of a series of profile grids with arbitrary space positions. Close to those listed is also the problem of flow around aircraft with a complex structure of the components, etc. A characteristic feature of the equation system associated with the problem in question is its block structure, each block consisting of elements relevant to only one of the bodies in the flow. Taking an advantage of this property, we present an iterative procedure solving such a system.

The main idea of the procedure is the successive solution of the equations of each block, which is physically equivalent to solving the problem of flow about isolated bodies in a perturbed flow. In what follows we prove that if the complete matrix of the equation system is strictly diagonally dominant, then the iterative process converges to the exact solution of the system, being started from an arbitrary initial approximation.

Our paper is arranged as follows. In Section II, we state the problem of flow past the blades of the regulating ring of a hydraulic turbine taking into account the mutual effects of a volute chamber, buttresses, and stator columns by restricting ourselves to the case of only 2-dimensional flow. Section III presents the block iterative algorithm solving the linear algebraic equation system with its convergence properties established. At last, Section IV provides a numerical example related to a real-life hydraulic turbine. Conclusions, Acknowledgement and References finish the manuscript.

\section{PROBLEM SPECIFICATION}

Following mainly the path of the authors' previous publications [16] and [17] assume that the fluid flow is stationary and potential within the 2-dimensional cross-cut of the mid-section of the volute chamber, stator, and the regulating ring of a turbine. Given the normal velocity $V_{n}=f\left(L_{0}\right)$ at the inlet section $L_{0}$ one should determine the distribution of velocity, $L_{0}$ pressure and hydrodynamic loads at the regulating ring blades, stator columns, buttresses and volute chamber, as well as at any point of a plane section across the flow (see Fig.1). In order to do

13 ${ }^{\text {th }}$ LACCEI Annual International Conference: "Engineering Education Facing the Grand Challenges, What Are We Doing?" 


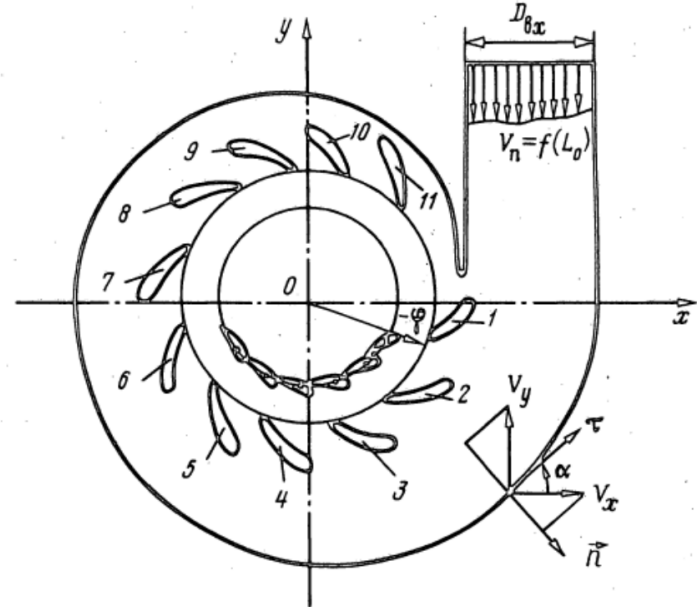

Fig.1. A hydraulic turbine cross-section

that, we use again ( $c f .$, [16] - [17]) the method of modeling a potential flow by a system of hydrodynamic singularities and replace the flow surface by a system of vortex contours. We model the impeller ring by a source and a vortex of intensities $Q$ and $\Gamma$, respectively, positioned in the center of the volute chamber, as determined by the residue of an analytic function at the singular point. Note that the normal velocity value $V_{n}$ is considered as given at any point of the contour $L$, which is the sum of all the turbine parts $L_{i}, i=1, \ldots, N$, including the regulating ring blades, stator columns, buttresses, and volute chamber. Therefore, we can write down the expression for $V_{n}$ in the following form:

$$
V_{n}=V_{x} \sin \alpha-V_{y} \cos \alpha=f(L),
$$

where $\alpha$ is the angle between the tangential (supporting) line to the contour $L$ and the $O x$ axis. Now we can obtain a singular integral equation for the vortex sheet density $\gamma(S)$. In equation (1), the velocity components with respect to the coordinate axes are calculated as follows:

$$
\begin{aligned}
& V_{x}=\frac{Q}{2 \pi} \frac{x-\xi}{R^{2}}-\frac{\Gamma}{2 \pi} \frac{y-\eta}{R^{2}}-\frac{1}{2 \pi} \oint_{L} \gamma(S) \frac{y-\eta}{R^{2}} d S+ \\
& +\frac{1}{2 \pi} \oint_{L} q(S) \frac{x-\xi}{R^{2}} d S,
\end{aligned}
$$

and

$$
\begin{aligned}
& V_{y}=\frac{Q}{2 \pi} \frac{y-\eta}{R^{2}}+\frac{\Gamma}{2 \pi} \frac{x-\xi}{R^{2}}+\frac{1}{2 \pi} \oint_{L} \gamma(S) \frac{x-\xi}{R^{2}} d S+ \\
& +\frac{1}{2 \pi} \oint_{L} q(S) \frac{y-\eta}{R^{2}} d S .
\end{aligned}
$$

Here, $x$ and $y$ are the Cartesian coordinates of the points where the velocity is computed, while $\xi$ and $\eta$ are those of the points at which hydrodynamic singularities are positioned, while $R^{2}=(x-\xi)^{2}+(y-\eta)^{2}$.

Now applying the method of discrete hydrodynamic singularities (cf., [8], [13]), which is also well-grounded in [12], one reduces equations (1) - (3) to the following system of linear algebraic equations:

$$
\begin{aligned}
& -\sum_{j=1}^{N} \gamma_{j} \frac{y_{i}-\eta_{j}}{R_{i j}^{2}} \sin \alpha_{i}-\sum_{j=1}^{N} \gamma_{j} \frac{x_{i}-\xi_{j}}{R_{i j}^{2}} \cos \alpha_{i} \\
& -\frac{\Gamma}{R_{i 0}^{2}}\left(y_{i}-y_{0}\right) \sin \alpha_{i}--\frac{\Gamma}{R_{i 0}^{2}}\left(x_{i}-x_{0}\right) \cos \alpha_{i}= \\
& =-\sum_{k=1}^{M} q_{k} \frac{x_{i}-\xi_{k}}{R_{i k}^{2}} \sin \alpha_{i}+\sum_{k=1}^{M} q_{k} \frac{y_{i}-\eta_{k}}{R_{i k}^{2}} \cos \alpha_{i}- \\
& -\frac{Q}{R_{i 0}^{2}}\left(x_{i}-x_{0}\right) \sin \alpha_{i}-\frac{Q}{R_{i 0}^{2}}\left(y_{i}-y_{0}\right) \cos \alpha_{i}+\pi V_{n}, \\
& i=1,2, \ldots, N .
\end{aligned}
$$

Here, $N$ is the number of computation points and $M$ is the number of discrete sources modeling the normal velocity profile at the inlet section $L_{0}$, while $x_{0}$ and $y_{0}$ are the coordinates of the point at which the source $Q$ and the vortex $\Gamma$ are located. The velocity of the flow is zero $\left(V_{n}=0\right)$ on the volute chamber surface, while $V_{n}=f\left(L_{0}\right)$ at each point of the inlet section. Finally, the following integral equations must be valid in the context of the considered problem:

$$
Q=\int_{L_{0}} V_{n} d S, \quad \sum_{i=1}^{N} \int_{L_{i}} \gamma_{i} d S_{i}+\Gamma=0 .
$$

The existence and uniqueness of the solution to the latter equations are ensured by the requirement that the ChaplyginZhukovskii postulate be true for the flow considered, in one of the forms usual for the hydraulic machine in question. Having solved equations (4) for $\gamma_{j}=\gamma_{j}(S), j=1, \ldots, N$, and $\Gamma$, one can determine the velocity components at any point $(x, y)$ using equations (2) and (3). The values of the dimensionless pressure $\bar{P}$, the tangential force value $c_{x}$ and that of the normal force $c_{y}$ at a chord of the unit length of each element $L_{i}, i=1, \ldots, N$, the moment quotients $c_{m}$ (with respect to the inlet edge of the body) and $c_{0}$ (with respect to the rotation axis of the regulating ring blade) are calculated by the formulas

$$
\begin{gathered}
\bar{P}=2 \frac{P_{\text {in }}-P}{\rho V_{\text {in }}^{2}}=1-\frac{V^{2}}{V_{\text {in }}^{2}}, \\
c_{x}=-\oint_{L_{i}} P \sin \alpha d S, \quad c_{y}=-\oint_{L_{i}} P \cos \alpha d S, \\
c_{m}=-\oint_{L_{i}}(x P \cos \alpha+y P \sin \alpha) d S,
\end{gathered}
$$

$1^{\text {th }}$ LACCEI Annual International Conference: “Engineering Education Facing the Grand Challenges, What Are We Doing?" 


$$
c_{0}=c_{m}+c_{y} x_{c}+c_{x} y_{c} .
$$

Here, $x_{c}$ and $y_{c}$ are the coordinates of the rotation axis of the regulating ring blade in a dimensionless coordinate system, the $O x$ axis of which coincides with the chord, and the origin is at the inlet rim; $P_{i n}$ and $V_{i n}$ are the pressure and velocity values, respectively, at the inlet section. Numerical solutions of the system of equations (1) - (7) is obtained through the standard discretization procedure (cf.,[16] - [18]) and then solving the (linear) system of equations (4).

\section{SOLUTION OF THE LINEAR SYSTEM}

In order to solve the system of linear equations (4) we propose a modification of the simple iteration and/or GaussSeidel methods and prove their convergence for the problem in question. First, we consider the following $m \times n$-matrix $A$ (here, we assume that $m \leq n$ ):

$$
A=\left(\begin{array}{rrrrr|rrr}
a_{11} & \varepsilon_{12} & \varepsilon_{13} & \ldots & \varepsilon_{1 m} & \varepsilon_{1, m+1} & \ldots & \varepsilon_{1 n} \\
-a_{21} & a_{22} & \varepsilon_{23} & \ldots & \varepsilon_{2 m} & \varepsilon_{2, m+1} & \ldots & \varepsilon_{2 n} \\
\varepsilon_{31} & -a_{32} & a_{33} & \ldots & \varepsilon_{3 m} & \varepsilon_{3, m+1} & \ldots & \varepsilon_{3 n} \\
\vdots & \vdots & \ddots & \ddots & \vdots & \vdots & \vdots \\
\varepsilon_{m 1} & \varepsilon_{m 2} & \ldots & -a_{m, m-1} & a_{m m} & \varepsilon_{m, m+1} & \ldots & \varepsilon_{m n}
\end{array}\right) .(8)
$$

By a specially arranged discretization procedure we can guarantee that the matrix entries boast the following properties:

1) The diagonal entries are positive $\left(a_{i i}>0, i=1, \ldots, m\right)$, while the sub-diagonal ones are, vice versa, negative $\left(-a_{i, i-1}<0, i=2, \ldots, m\right)$.

2) The diagonal and sub-diagonal entries' absolute values are much greater than those of the rest of the matrix' elements. More exactly, let the inequalities listed below hold:

$$
\left\{\begin{array}{l}
\left|a_{11}\right|>\sum_{j=2}^{n}\left|\varepsilon_{1 j}\right|, \\
\left|a_{22}+\varepsilon_{12}\right|>\left|a_{11}-a_{21}\right|+\sum_{j=3}^{n}\left|\varepsilon_{1 j}+\varepsilon_{2 j}\right|, \\
\left|a_{33}+\varepsilon_{13}+\varepsilon_{23}\right|>\left|a_{11}-a_{21}+\varepsilon_{31}\right|+\left|a_{22}-a_{32}+\varepsilon_{12}\right|+ \\
+\sum_{j=4}^{n}\left|\varepsilon_{1 j}+\varepsilon_{2 j}+\varepsilon_{3 j}\right|, \\
\vdots \\
\left|\begin{array}{l}
a_{m m}+\sum_{k=1}^{m-1} \varepsilon_{k m}|>| a_{11}-a_{21}+\sum_{k=3}^{m} \varepsilon_{k 1} \mid+ \\
+\left|a_{22}-a_{32}+\varepsilon_{12}+\sum_{k=4}^{m} \varepsilon_{k 2}\right|+\ldots+ \\
+\left|a_{m-1, m-1}-a_{m, m-1}+\sum_{k=1}^{m-2} \varepsilon_{k, m-1}\right|+\sum_{j=m+1}^{n}\left|\sum_{k=1}^{m} \varepsilon_{k j}\right|
\end{array}\right|
\end{array}\right.
$$

In our previous papers [16] and [17], property (9) was called the concealed strict diagonal dominance (CSDD) in matrix $A$. Below we will show why this name (or, the implicit strict diagonal dominance, ISDD) is appropriate, and how it helps one prove the convergence of the simple iteration and/or Gauss-Seidel methods solving the linear equation $A x=b$ for an arbitrary $b \in R^{m}$.

First of all, we note that property (9) is an extension of the classical diagonal dominance. Indeed, in the particular case when $a_{11}=a_{21}=a_{22}=\cdots=a_{m, m-1}=a_{m m}=a>0$, matrix $A$ is evidently not strictly diagonally dominant even if all $\varepsilon_{i j}=0$. However, property (9), which in this particular case reduces to

$$
\left\{\begin{array}{l}
|a|>\sum_{j=2}^{n}\left|\varepsilon_{1 j}\right|, \\
\left|a+\varepsilon_{12}\right|>\sum_{j=3}^{n}\left|\varepsilon_{1 j}+\varepsilon_{2 j}\right|, \\
\left|a+\varepsilon_{13}+\varepsilon_{23}\right|>\left|\varepsilon_{31}\right|+\left|\varepsilon_{12}\right|+\sum_{j=4}^{n} \varepsilon_{1 j}+\varepsilon_{2 j}+\varepsilon_{3 j} \mid, \\
\vdots \\
\left|a+\sum_{k=1}^{m-1} \varepsilon_{k m}\right|>\left|\sum_{k=3}^{m} \varepsilon_{k 1}\right|+\left|\varepsilon_{12}+\sum_{k=4}^{m} \varepsilon_{k 2}\right|+\ldots+\left|\sum_{k=1}^{m-2} \varepsilon_{k, m-1}\right|+ \\
+\sum_{j=m+1}^{n}\left|\sum_{k=1}^{m} \varepsilon_{k j}\right|,
\end{array}\right.
$$

clearly holds if the absolute value of the diagonal entries $a$ is much larger than those of the non-diagonal elements $\varepsilon_{i j}$. For instance, property (10) is valid when 


$$
|a|>\sum_{i=1}^{m-1} \sum_{j=i+1}^{m}\left|\varepsilon_{i j}\right|+\sum_{j=1}^{m-2} \sum_{i=j+2}^{m}\left|\varepsilon_{i j}\right|+\sum_{i=1}^{m} \sum_{j=m+1}^{n}\left|\varepsilon_{i j}\right|
$$

holds.

Now we will show that the ISDD property (9), on the other hand, implies the (strict) diagonal dominance of another matrix $D$ related to the original matrix $A$ in such a way that the systems $A x=b$ and $D x=g$ are equivalent, i.e., their solutions coincide. In order to do that, denote by $B$ the principal square submatrix of matrix $A$ :

$$
B=\left(\begin{array}{rrrrr}
a_{11} & \varepsilon_{12} & \varepsilon_{13} & \ldots & \varepsilon_{1 m} \\
-a_{21} & a_{22} & \varepsilon_{23} & \ldots & \varepsilon_{2 m} \\
\varepsilon_{31} & -a_{32} & a_{33} & \ldots & \varepsilon_{3 m} \\
\vdots & \vdots & \ddots & \ddots & \vdots \\
\varepsilon_{m 1} & \varepsilon_{m 2} & \ldots & -a_{m, m-1} & a_{m m}
\end{array}\right)
$$

In [17], the following result was established:

Theorem 1. As explained in [17]. If an $m \times n-$ matrix $A$ having structure (8) satisfies condition (9), then its principal square submatrix $B$ has the inverse $B^{-1}$. Furthermore, the matrix

$$
D:=B^{-1} A=\left(\begin{array}{rrrrr|rrr}
1 & 0 & 0 & \ldots & 0 & d_{1, m+1} & \ldots & d_{1 n} \\
0 & 1 & 0 & \ldots & 0 & d_{2, m+1} & \ldots & d_{2 n} \\
0 & 0 & 1 & \ldots & 0 & d_{3, m+1} & \ldots & d_{3 n} \\
\vdots & \vdots & \vdots & \ddots & \vdots & \vdots & \ldots & \vdots \\
0 & 0 & 0 & \ldots & 1 & d_{m, m+1} & \ldots & d_{m n}
\end{array}\right)
$$

is strictly diagonally dominant (in the classical sense), that is,

$$
\sum_{j=m+1}^{n}\left|d_{i j}\right|<1 \text {, for all } i=1,2, \ldots, m \text {. }
$$

When proving Theorem 1 in [17], it was demonstrated that if matrix $A$ has the ISDD property (9) then an auxiliary matrix $A_{1}$ generated by a special transformation of $A$ boasts the (classical) strict diagonal dominance. The said transformation is described as follows: to obtain the matrix $A_{1}$, one adds the top row (row 1 ) of matrix $A$ to its row 2; then the newly updated row 2 is added to 3 of the same matrix, and so on. The sum of all rows 1 through $(m-1)$ is finally added to the bottom row $m$ of the original matrix $A$, thus resulting in the auxiliary matrix $A_{1}$. Now it is not difficult to see that the transformation in question can be represented in the matrix form as shown below:

$$
A_{1}=L A \text {, }
$$

where $L$ is the lower triangular (square and invertible) matrix

$$
L=\left(\begin{array}{ccccc}
1 & 0 & 0 & \ldots & 0 \\
1 & 1 & 0 & \ldots & 0 \\
1 & 1 & 1 & \ldots & 0 \\
\vdots & \vdots & \vdots & \ddots & \vdots \\
1 & 1 & 1 & \ldots & 1
\end{array}\right),
$$

the inverse of which can be found explicitly as follows:

$$
L^{-1}=\left(\begin{array}{rrrrr}
1 & 0 & 0 & \ldots & 0 \\
-1 & 1 & 0 & \ldots & 0 \\
0 & -1 & 1 & \ldots & 0 \\
\vdots & \vdots & \ddots & \ddots & 0 \\
0 & 0 & 0 & -1 & 1
\end{array}\right)
$$

All those results can be collected in the lemma below.

Lemma 2. The inverse to the lower triangular matrix $L$ defined by (16) is the lower bi-diagonal matrix $L^{-1}$ shown in (17), whose entries are determined by the explicit formula:

$$
l_{i j}=\left\{\begin{array}{rr}
1, & \text { if } i=j ; \\
-1, & \text { if } i=j+1, j=1, \ldots, m-1 ; \\
0, & \text { otherwise. }
\end{array}\right.
$$

Proof. The proof is straightforward: we left-multiply matrix $L$ by the matrix (17) - (18). Indeed, $(i, j)$ - entries of the product $L^{-1} L$ are defined by the inner product of row $i$ of matrix $L^{-1}$ by column $j$ of matrix $L$. Now since column $j$ of matrix $L$ contains zero entries from the top row 1 to row $j-1$, and has units from row $j$ downward, while row $j$ of matrix $L^{-1}$ comprises zero entries except for columns $j-1$ and $j$ where it contains $(-1)$ and 1 , respectively, then it is clear that the $j$-th diagonal entry of $L^{-1} L$ is 1 for all $j=1, \ldots, m$. Similarly, it is easy to see that the non-diagonal entries $(i, j), i \neq j$, of the product $L^{-1} L$ are all zero, because both non-zero entries of row $i$ of matrix $L^{-1}$, i.e., $(-1)$ and 1 , are multiplied by zero (when $i<j$ ) or by $1 \quad$ (if $i>j$ ). The sum of these two products equals zero in both cases, which completes the proof: indeed, the above arguments allow one to conclude that the product $L^{-1} L$ is the $m \times m$ identity matrix $I$.

Now we are in a position to formulate and prove the following interesting result for the linear systems of equations and their (rectangular) matrices.

Theorem 3. There exists a one-to-one correspondence between the $m \times n-$ matrices $A(m \leq n)$ with the implicit strict diagonal dominance (ISDD) and the $m \times n-$ matrices $A_{1}$ boasting the (classical) strict diagonal dominance (SDD). 
Moreover, this correspondence is established with leftmultiplying the matrix $A$ by the lower triangular matrices $L$ and $L^{-1}$ defined by (16) and (17) - (18), respectively. Finally, each linear equation $A x=b$ with matrix $A$ having ISDD has a unique solution for any right-hand side vector $b \in R^{m}$.

Proof. Relationships given by equation (15) and its inverse

$$
L^{-1} A_{1}=A \text {, }
$$

immediately establish the desired one-to-one correspondence among the ISDD-matrices $A$ and SDD- matrices $A_{1}$.In order to prove the last assertion of the theorem, it is enough to leftmultiply the linear equation $A x=b$ by the transition matrix $L$. Now since the resulting equivalent system $L A x=L b$ can be rewritten as $A_{1} x=g$ with $g=L b$, the expected result follows directly. Indeed, matrix $A_{1}$ having the strict diagonal dominance implies the existence of exactly one solution to the equation $A_{1} x=g$, which also solves the original system $A x=b$.

\section{Applications to HydraUlic Machines}

Coming back to the hydraulic machine problem described by equations (1) - (3) consider equation (4) as a linear system to be solved with the aid of simple iteration methods. First, let us rewrite system (4) in the matrix form as

$$
H u=\beta \text {, }
$$

where $u=(\xi, \eta) \in R^{T}, \beta \in R^{T}$ with $T=2 N+2 M$, and $H$ is a large-scale (square) matrix of the block structure:

$$
H=\left(\begin{array}{rrrr}
H_{11} & H_{12} & \ldots & H_{1 s} \\
H_{21} & H_{22} & \ldots & H_{2 s} \\
\vdots & \vdots & \ddots & \vdots \\
H_{s 1} & H_{s 2} & \ldots & H_{s s}
\end{array}\right) .
$$

Here, every block $H_{i j}, i, j=1, \ldots, s$, is an $\left(m_{i} \times m_{j}\right)$-matrix with $m_{1}+m_{2}+\cdots+m_{s}=T$. Suppose further that each horizontal "layer"

$$
\left[\begin{array}{lllll}
H_{i 1} & H_{i 2} \ldots & H_{i i} \ldots & H_{i s}
\end{array}\right], i=1,2, \ldots, s,
$$

has the structure described in equation (8) and satisfies the ISDD condition (9) with the square block $H_{i i}$ playing the role of the principal square submatrix $B$. It follows then from Theorem 1 (from [17]) that each matrix $H_{i i}, i=1,2, \ldots, s$, is invertible. Hence, one can apply the following algorithm to solve problem of equation (20) numerically.

First, we fulfill a preliminary step consisting in leftmultiplying matrix $H$ by the block- diagonal matrix

$$
H_{d}=\left(\begin{array}{rrrr}
H_{11}^{-1} & 0 & \ldots & 0 \\
0 & H_{22}^{-1} & \ldots & 0 \\
\vdots & \vdots & \ddots & \vdots \\
0 & 0 & \ldots & H_{s s}^{-1}
\end{array}\right) .
$$

In the practical implementations of the algorithm, of course, this step is made by the standard Gaussian elimination process.

After having done that, system of equation (20) can be rewritten in the equivalent form

$$
F u=\delta,
$$

where $\delta=H_{d} \beta$, and the matrix $F=H_{d} H$ has the following block structure

$$
F=\left(\begin{array}{cccc}
I_{1} & H_{11}^{-1} H_{12} & \ldots & H_{11}^{-1} H_{1 s} \\
H_{22}^{-1} H_{21} & I_{2} & \ldots & H_{22}^{-1} H_{2 s} \\
\vdots & \vdots & \ddots & \vdots \\
H_{s s}^{-1} H_{s 1} & H_{s s}^{-1} H_{s 2} & \ldots & I_{s}
\end{array}\right) .
$$

Here, $I_{k}$ is the $\left(m_{k} \times m_{k}\right)$ unit matrix, $k=1,2, \ldots, s$. Then we reformulate equation (24) as

$$
u=G u+\delta
$$

with the block-structured matrix

$$
G=\left(\begin{array}{cccc}
0 & -H_{11}^{-1} H_{12} & \ldots & -H_{11}^{-1} H_{1 s} \\
-H_{22}^{-1} H_{21} & 0 & \ldots & -H_{22}^{-1} H_{2 s} \\
\vdots & \vdots & \ddots & \vdots \\
-H_{s s}^{-1} H_{s 1} & -H_{s s}^{-1} H_{s 2} & \ldots & 0
\end{array}\right),
$$

which allows one to apply the simple iteration (Jacobi) method to solve it.

The Jacobi algorithm consists in calculating the successful iterations as follows:

$$
\left\{\begin{array}{l}
u^{(t+1)}:=G u^{(t)}+\delta, \quad t=0,1, \ldots ; \\
u^{(0)}:=\delta .
\end{array}\right.
$$

The approximations $\left\{u^{(t)}\right\}_{t=0}^{\infty}$ converge to the (unique) solution $u$ of the linear equation (20) since all the eigenvalues $\lambda_{k}=\lambda_{k}(G)$ of matrix $G$ belong to the open interval $(-1,1)$, i.e., $\left|\lambda_{k}(G)\right|<1$. The latter inequality follows from estimates (14) from Theorem 1 [17] and the well-known Gershgorin circle theorem (cf., [14] and [15]).

By the way, with all the details above, we have just proven the following result. 
Theorem 4. The sequence of approximations $\left\{u^{(t)}\right\}_{t=0}^{\infty}$ generated by the simple iteration process (27) - (28) converges to the unique solution $u$ of the linear system of equations (20) starting from an arbitrary initial approximation $u^{(0)} \in R^{T}$.

Remark 1. As is well-known ( $c f .$, [14] and [15]), both the simple iteration method and the Gauss-Seidel algorithm to solve linear equations boast convergence under the same conditions. Therefore, instead of the simple iteration algorithm described by equation (28), in order to solve the reduced linear system (26) one can apply the iteration formulas of the GaussSeidel method:

$$
\left\{\begin{array}{l}
u^{(t+1)}:=G \tilde{u}^{(t)}+\delta, \quad t=0,1, \ldots ; \\
u^{(0)}:=\delta
\end{array}\right.
$$

Here, the only difference between the update formulas (28) and (29) is in that the latter makes use of the partially updated previous iteration vector $\tilde{u}^{(t)}$ instead of the unchanged vector $u^{(t)}$ used in the former. See the details in [14] and [15].

Based upon Remark 1, one can easily establish the following result.

Theorem 5. The sequence of approximations $\left\{u^{(t)}\right\}_{t=0}^{\infty}$ generated by the Gauss-Seidel algorithm (27) and (29) converges to the unique solution $u$ of the linear system of equations (20) starting from an arbitrary initial approximation $u^{(0)} \in R^{T}$.

\section{A NumericAl EXAMPLE}

The above-described algorithms solving linear equation system (20) were applied to compute the hydrodynamic loads on the blades of a hydraulic turbine regulating ring using a volute chamber and stator columns, by the method of discrete vortices [12]. The following numbers of computation points were used: 90 at the volute chamber, and 31 each on each stator column and regulating ring blade.

The experiments were run on an Intel_Core 2 Quad $^{\mathrm{TM}} 2.66$ $\mathrm{MHz}$ running Windows Vista ${ }^{\mathrm{TM}}$ Home Premium, and using Matlab $^{\circledR}$ R2008b.

Computations were performed for a set of real generators, the number of stator columns varying from 11 to 20 and the number of regulating ring blades varying from 20 to 24 .
Computation accuracy $\varepsilon=0.1$ was determined by the magnitude of the circulation

$$
\Gamma=\int_{L} V d S,
$$

where $V$ is the flow velocity at each profile $L_{i}$. The positions of the stator columns and regulating ring blades are shown in Fig.1.

Table 1 below demonstrates the computed moment quotients $c_{m}$ with respect to the rotation axis of the regulating ring blades and compares them to the experimental data concerning the same moment quotients $c_{m}$ obtained for the generator produced in Kharkiv, Ukraine, for "Piedra del Aguila" hydroelectric power plant in Neuquén, Argentina. The specified accuracy of $\varepsilon=10 \%$ at each of the elements was achieved after 12 to 20 iterations.

In order to ensure condition (10) to be satisfied for matrix $A$ of linear equation (8), all the matrix coefficients were computed after having positioned the hydrodynamic singularity $\gamma(S)$ at the distance of $0.1 \Delta S$ from the computation point, as is recommended by the discrete vortex method [12].

A test verification of this discretization algorithm in the case of plane ideal liquid flow around a cylinder demonstrated a good agreement between the numerical results and the analytical solution, which justifies the use of the algorithm for practical problems.

\begin{tabular}{|c|c|c|c|c|c|c|}
\hline \multicolumn{7}{|c|}{ RESULTS OF NUMERICAL EXPERIMENTS } \\
\hline & \multicolumn{6}{|c|}{ Number of blades } \\
\hline & 3 & 4 & 5 & 9 & 10 & 11 \\
\hline Computed $c_{m}$ & 0.23 & 0.17 & 0.22 & 0.065 & 0.13 & 0.08 \\
\hline $\begin{array}{l}\text { Experimental } \\
c_{m}\end{array}$ & 0.25 & 0.17 & 0.24 & 0.070 & 0.11 & 0.08 \\
\hline & \multicolumn{6}{|c|}{ Number of blades } \\
\hline & 15 & 16 & 17 & 20 & 21 & 22 \\
\hline Computed $c_{m}$ & 0.22 & 0.205 & 0.249 & 0.315 & 0.069 & 0.12 \\
\hline $\begin{array}{l}\text { Experimental } \\
c_{m}\end{array}$ & 0.23 & 0.210 & 0.240 & 0.320 & 0.060 & 0.12 \\
\hline
\end{tabular}

TABLE I

Our experience in the use of the numerical tools for computing the parameters of ideal liquid flow around the regulating ring blades of a hydraulic turbine, as well as the satisfactory agreement between the numerical and experimental values of the turning moment $c_{m_{0}}$ on the blade confirm the reliability of the above algorithms when solving systems of linear equations arising in engineering problems. 


\section{CONCLUSIONS}

In this paper, making use of the method of discrete hydrodynamic singularity and a condition on the velocity normal component at the flow's boundary, we reduce the problem of ideal liquid flow around arbitrarily positioned bodies to solving a system of linear algebraic equations with a block structure. We apply the discretization approach, which provides for an implicit strict diagonal dominance (ISDD) in the system's matrix. We establish the one-to-one correspondence between the class of matrices having the ISDD property and the classical strict diagonal dominant matrices. Convergence of a block Jacobi iteration method to the exact solution of the thus obtained equation system is established. The algorithm's efficiency is confirmed by a sample computing of hydrodynamic charges on the hydraulic turbine's regulating ring blades ( $c f$. [17]).

Since the convergence conditions for the Gauss-Seidel iteration method coincide with those for the Jacobi algorithm, one can apply the Gauss-Seidel iterations to find the hydraulic machine hydrodynamic loads and the liquid velocity on the turbine parts as well.

\section{ACKNOWLEDGMENT}

The research activity of the first and third authors was financially supported by the R\&D Department of the Tecnológico de Monterrey (ITESM), Campus Monterrey, and by the SEP-CONACYT projects CB-2013-01-221676. Also, the second author was supported by the PAICYT project No. CE250-09 and by the SEP-CONACYT project CB-2009-01127691.

The authors also would like to express their profound gratitude to the three anonymous referees whose valuable critical comments and suggestions have helped improve the presentation.

\section{REFERENCES}

[1] M.A. Lavrent'ev, Ya.I. Sekerzh-Zen'kovich and V.M. Shepelev,On the theory of biplane wing frames, CAGI Proceedings, Moscow, 153, 1935 (in Russian).

[2] S.G. Mikhlin, Integral Equations and Applications to Problems in Mechanics, Mathematical Physics and Technology, Gostekhteorizdat, Moscow-Leningrad, 1949 (in Russian).

[3] G.Yu. Stepanov, Hydrodynamics of Turbine Grids, Fizmatgiz, Moscow, 1961 (in Russian).

[4] L.I. Sedov, Planar Problems of Hydrodynamics and Aerodynamics, Nauka, Moscow, 1966 (in Russian).

[5] L.Ya. Kazachkov, "Non-stationary flow past a multiple two-dimensional turbine grid in a layer of variable thickness," Energeticheskoe Mashinostroenie, vol. 6, pp. 18-23, 1970 (in Russian).
[6] V.E. Saren, "On the hydrodynamic interaction of profile grids in a nonstationary flow," Izvestia Akademii Nauk S.S.S.R., Mekhanika Zhidkosti i Gaza, vol. 4, pp. 75- 84, 1971 (in Russian).

[7] G.S. Samoilovich, The Excitation of Turbine Blade Vibrations, Mashinostroyeniye, Moscow, 1975 (in Russian).

[8] S.M. Belotserkovskii and M.I. Nisht, Separated and Non-Separated Flow past Thin Aerofoils by Ideal Liquid, Nauka, Moscow, 1978 (in Russian).

[9] V.I. Gnesin, "Aerodynamic interaction of moving profile grids," Izvestia Akademii Nauk S.S.S.R., Energetika i Transport, vol.1, pp. 102- 109, 1982 (in Russian).

[10] V.A.Yudin, "Non-stationary aerodynamic reactions at the profiles of two grids in relative motion," CAGI Proceedings, Moscow, vol. 1064, pp. 6270, 1983 (in Russian).

[11] V.I. Gnesin and S.V. Yershov, "Calculation of the aerodynamic interactions of spatial grids with an arbitrary blade number ratio," Problemy Prochnosti, vol. 18, pp. 84- 89, 1983 (in Russian).

[12] S.M. Belotserkovskii and I.K. Lifanov, Numerical Methods Solving Singular Integral Equations and Their Application to Aerodynamics, Elasticity Theory and Electrodynamics, Nauka, Moscow, 1985 (in Russian).

[13] S.M. Belotserkovskii, V.N. Kotovskii, M.I. Nisht and R.M. Fedorov, Mathematical Modelling of Plane-Parallel Separated Flow past Solids, Nauka, Moscow, 1988 (in Russian).

[14] R.S. Varga, Geršgorin and His Circles. Berlin: Springer-Verlag, 2004.

[15] G.H. Golub, C.F. Van Loan, Matrix Computations. Baltimore: Johns Hopkins University Press, 1996.

[16] V.V. Kalashnikov and S.D. Kostornoi, "A numerical method solving the problem of ideal liquid flow around arbitrarily positioned bodies," Computational Mathematics and Mathematical Physics, vol. 32, No. 6, pp. 837-844, 1992.

[17] V.V. Kalashnikov and S.D. Kostornoi, "Problem of ideal liquid flowing around arbitrarily positioned bodies: Numerical solution and application to hydraulic machines." - In: Gary C. Cohen (Ed.), Proceedings of the $3^{\text {rd }}$ International Conference on Mathematical and Numerical Aspects of Wave Propagation, La Napoule, France, 1995, pp. 372- 383.

[18] S.D. Kostornoi and N.S. Martynova, "Selection of a model for the liquid flow when developing a hydraulic machine with blades," Visnyk SumDU, Ser. Technical Sciences, vol. 2, pp. 18- 28, 2012 (in Russian). 